\title{
Validez Facial del Instrumento de Medición de Hábitos de Vida (MHAVIDA) en Personas con Discapacidad de 5-13 Años
}

\author{
Facial Validity of the Measuring Instrument of Habits of \\ Life (MHAVIDA) in Disability Persons Between 5-13 Years
}

Olga Santamaría Pinzón ${ }^{1}$

Universidad de Santander

Chantal Camden ${ }^{2}$

Université de Sherbrooke

Adriana Angarita Fonseca ${ }^{3}$

Universidad de Santander

\section{RESUMEN}

Introducción: La Clasificación Internacional del Funcionamiento (CIF) ha definido la discapacidad como un término que incluye deficiencias, limitaciones en la actividad y restricciones en la participación. Objetivo: Establecer la validez facial del instrumento de medición de hábitos de vida (MHAVIDA), que evalúa la participación social en niños y niñas de entre 5 y 13 años de edad que se encuentran en Condición de Discapacidad (CD). Materiales y métodos: Se realizó un estudio de evaluación de pruebas diagnósticas, así como la adaptación cultural de los instrumentos con 6 madres de niños y niñas sin condición de discapacidad (CD). A su vez, la validez facial fue evaluada por 19 madres de niños y niñas en CD, a quienes se les aplicó el instrumento mediante entrevista, realizada por dos estudiantes de último año de Fisioterapia. Resultados: El instrumento MHAVIDA para el rango de 5 a 13 años de edad, que evalúala participación social fue traducido oficialmente, y adaptado al lenguaje local con preguntas comprensibles, claras y fáciles de responder. Se encontraron palabras o preguntas confusas principalmente en las categorías educación y nutrición del instrumento que fueron modificadas y, finalmente, se obtuvo un instrumento con validez facial y adaptabilidad cultural pertinente al contexto. Conclusión: Se obtuvo un instrumento útil para evaluar la participación social a partir de los hábitos de vida de la población de 5 a 13 años con discapacidad, lo cual proporciona una base sólida para mejorar las intervenciones en rehabilitación.

Palabras Clave: participación social, evaluación de la discapacidad, niños con discapacidad (Fuente: DeCS).

\begin{abstract}
Introduction: The International Classification of Functioning (ICF) has defined disability as a term that includes impairments, activity limitations and restrictions. Objective: To establish the facial validity of the instruments Assessment of Life Habits (LIFE-H) for disabled children between 5 and13 years old. Materials and methods: a study was carried out on the assesment of diagnostic tests, as well as the cultural adaptation of the instruments using 6 mothers of children without disabilities (CD) as a reference. At the same time, the validity was assessed by 19 mothers of boys and girls in CD who were assessed through an interview done by two last year physiotherapy students. Results: The LIFE-H for age 5-13 years old, in order to assess social participation were officially translated, adapted to local language with comprehensible, clear, and easy to answer questions. We obtained an instrument with facial validity and relevant cultural adaptability to the context. We found confusing words or questions in education and nutrition categories in the LIFE-H for 5-13 years old that were modified. Conclusion: We obtained a useful tool to measure social participation from the habits of children with disabilities, which provides a sound basis for improving interventions in rehabilitation. It is suggested to continue the evaluating the reliability of this instrument.
\end{abstract}

Keywords: social participation, disability evaluation, disabled children (Source: MeSH, NLM).

\footnotetext{
${ }^{1}$ Fisioterapeuta. Especialista en Docencia Universitaria. Correo electrónico: olgasan8@hotmail.com

${ }^{2}$ Fisioterapeuta, Phd (c) Docente Université de Sherbrooke.

${ }^{3}$ Fisioterapeuta. Magister en Epidemiología. Correo electrónico: adriangarita@udes.edu.co
} 


\section{Introducción}

La Clasificación Internacional del Funcionamiento, de la Discapacidad y de la Salud (CIF) (1) ha definido la discapacidad como un término genérico que incluye déficits, limitaciones en la actividad y restricciones en la participación. Concretamente, en Colombia, los resultados del Censo 2005 muestran que el 6,4\% de los colombianos presentan al menos una limitación (2).

Ahora bien, es claro que la discapacidad genera impacto en la adaptación, rehabilitación e integración social de las personas con esta condición, lo cual ha convertido en una preocupación importante en contextos clínicos y de investigación (3). Adicionalmente, ha surgido la necesidad institucional y social de usar el mismo lenguaje y un modelo común a la hora de abordar a la persona en condición de discapacidad (CD) (1).

Este abordaje incluye la valoración de la participación, que según la CIF es el acto de involucrarse en una situación vital y está constituida por nueve dominios (1). Actualmente, existe controversia sobre cuáles dominios de la CIF deben incluirse al evaluar la participación y si las actividades y la participación son aspectos distintos (4).

Por otro lado, en el Modelo de Creación del Proceso de Discapacidad (MCPD) propuesto por Fougeyrollas, el concepto de participación fue operacionalizado mediante la evaluación de los hábitos de vida (5), entendidos como los roles sociales y las actividades diarias que aseguran el desarrollo y la supervivencia de la persona en la sociedad a lo largo de su vida (3). Éstos son evaluados por medio del instrumento de Medición de Hábitos de Vida (MHAVIDA), que se aplica mediante entrevista a las madres o cuidadores del niño o niña en Condición de Discapacidad (CD). Este instrumento comprende las 12 dimensiones de la participación del MCPD: nutrición, condición corporal, cuidado personal, comunicación, vivienda, desplazamientos, responsabilidades, relaciones interpersonales, vida comunitaria, educación, recreación y trabajo (3).

Las propiedades psicométricas de este instrumento han sido previamente evaluadas en otros contextos. La reproducibilidad prueba-reprueba del instrumento fue evaluada en pacientes con lesión medular, obteniéndose un Coeficiente de Correlación Intraclase (CCI) de 0,67en niños, y en adultos, de 0,83(5). Otro estudio en niños de 5-13 años mostró alta reproducibilidad, con CCI por encima de 0,78 para 10 de las 11 categorías evaluadas (6).

En la revisión de la literatura, no se encontraron estudios que evaluaran las propiedades psicométricas del instrumento MHAVIDA en población colombiana $\mathrm{y}$, dado que el instrumento fue desarrollado para una población culturalmente diferente y en otro idioma, con este trabajo se pretende evaluar su validez facial, que evalúa la participación social en niños y niñas en condición de discapacidad de 5 a 13 años, previa traducción y adaptación cultural.

\section{Materiales y Métodos}

Como ya se expresó, se realizó un estudio de evaluación de pruebas diagnósticas para evaluar la validez facial del instrumento, previa traducción y adaptación cultural (7). En lo que corresponde a la adaptación cultural, se seleccionaron 6 familiares o cuidadores de niños y niñas sin CD. En cambio, la validez facial fue realizada por los familiares o cuidadores de niños y niñas en $\mathrm{CD}$, excluyéndoselos familiares y /o cuidadores que no disponían de tiempo para el diligenciamiento del instrumento MHAVIDA.

El estudio se realizó en tres fases. La primera comprendió la traducción del instrumento MHAVIDA; la segunda, la adaptación cultural; y la tercera, la validez facial del instrumento.

La traducción oficial del instrumento del idioma Inglés al español fue realizada por intermedio del Centro de Idiomas de la Universidad de Santander (UDES), previa autorización de los creadores para su uso en investigación. Los ajustes necesarios se realizaron en colaboración con cuatro expertos en el área. Más en específico, un asesor internacional, quien dominaba ambos idiomas, inglés y español, realizó la traducción del inglés al español. De esta manera, se obtuvo la versión en español 1.0 del instrumento MHAVIDA.

En la segunda fase, se contactaron, inicialmente, seis familiares o cuidadores de niños y niñas sin CD de un hogar comunitario del Instituto Colombiano de Bienestar Familiar de la Comuna 2 de Bucaramanga, con el fin de conocer si para ellos la redacción y la extensión de las preguntas era la adecuada. Realizadas estas entrevistas, se procedió a revisar y a hacer las

Cienc. innov. salud. Junio 2013; 1 (1):18 - 26. Universidad Simón Bolívar (Col). ISSN: 2344-8636 http://portal.unisimonbolivar.edu.co:82/rdigital/innovacionsalud 
modificaciones pertinentes al instrumento para crear la versión en español 2.0 del instrumento MHAVIDA.

La nueva versión del instrumento MHAVIDA fue sometida, luego, a evaluación por 19 madres de niños y niñas en CD de la Fundación FANDIC.

Para la recolección de la información se citó con anterioridad a los encuestados a las instalaciones de la fundación FANDIC durante el segundo semestre de 2010. El día de la aplicación, se explicó el objetivo del estudio y la metodología como parte del proceso del consentimiento informado. Ya aceptada la participación de las madres, se procedió con la aplicación del formato, resolviendo dudas e inquietudes de las encuestadas. La entrevista individual fue realizada por dos estudiantes de último año de la carrera de Fisioterapia. Uno entrevistaba y diligenciaba el formato, y el otro registraba las preguntas, dudas, sugerencias o comentarios de la madre o cuidador durante la entrevista.

Las recomendaciones o dudas que surgieron de ambos procesos fueron resueltas por un miembro del equipo de creadores del instrumento MHAVIDA, versión canadiense $\mathrm{y}$, de este modo, se obtuvo la versión en español 3.0 del instrumento.

El instrumento MHAVIDA fue desarrollado con el objetivo de evaluar dos aspectos relacionados con la participación social de las personas con discapacidad: la forma de realizar las actividades que se llevan a cabo en la vida diaria (acostarse o salir de la cama, comer, etc.) y los roles sociales (comprar en la tienda, educación, etc.), independientemente del tipo de deficiencias subyacentes. El instrumento concebido para niños de 5-13 años consta de 64 preguntas agrupadas en 12 categorías de hábitos de vida (Tabla $1)$.

Tabla 1. Número de preguntas en las categorías de hábitos de vida para el instrumento MHAVIDA de 5-13 años

\begin{tabular}{clc}
\hline \multirow{2}{*}{ Actividades evaluadas } & \multicolumn{1}{c}{ Categoría de hábito de vida } & \multicolumn{1}{c}{ Instrumento MHAVIDA } \\
\cline { 3 - 3 } Actividad de la vida diaria & Para niños, niñas y jóvenes de 5-13 años \\
& Nutrición & 4 \\
& Condición Corporal & 4 \\
& Cuidado personal & 8 \\
& Comunicación & 8 \\
& Vivienda & 6 \\
& Desplazamientos & 4 \\
\hline & Responsabilidades & 7 \\
& Relaciones interpersonales & 6 \\
\multirow{5}{*}{ Roles sociales } & Vida comunitaria & 2 \\
& Educación & 6 \\
& Recreación & 8 \\
& Trabajo & 1 \\
\hline
\end{tabular}

El instrumento MHAVIDA permite documentar el nivel de logro de cada hábito de vida mediante una calificación de 0 a 9 , que depende del grado de dificultad en la realización y el tipo de ayuda requerida para lograr este objetivo. Es de resaltar que si el familiar o cuidador considera que un modo de vida específico no hace parte de la vida cotidiana o el estilo de vida de la persona en $\mathrm{CD}$, ésta debe marcarse entonces como no aplicable (Tabla 2). La calificación se puede llevar a una puntuación estándar mediante la aplicación de la siguiente fórmula: ( $\Sigma$ puntuación x 10) dividido por (número de hábitos de vida aplicables por 9). Por ejemplo, si al evaluar el hábito de vida Nutrición a un niño de 6 años en quien aplican todas las preguntas, y cada pregunta tiene una calificación de 5 , se tendría en el numerador: $(5+5+5+5) \times 10=200$ y en el denominador: 4 × $9=36$, siendo el puntaje del hábito de vida Nutrición de 5,6 (200/36). 
Tabla 2. Escala de calificación de los hábitos de vida

\begin{tabular}{cll}
\hline Puntuación & \multicolumn{1}{c}{ Nivel de dificultad } & \multicolumn{1}{c}{ Tipo de asistencia } \\
\hline $\mathbf{9}$ & Sin dificultad & Sin ayuda \\
$\mathbf{8}$ & Sin dificultad & Ayuda técnica \\
$\mathbf{7}$ & Con dificultad & Sin ayuda \\
$\mathbf{6}$ & Con dificultad & Adaptación o acondicionamiento \\
$\mathbf{5}$ & Sin dificultad & Ayuda humana adicional \\
$\mathbf{4}$ & Sin dificultad & Adaptación o acondicionamiento. Ayuda humana adicional \\
$\mathbf{3}$ & Con dificultad & Ayuda humana adicional \\
$\mathbf{2}$ & Con dificultad & Adaptación o acondicionamiento. Ayuda humana adicional \\
$\mathbf{1}$ & Realizado por Sustitución & \\
$\mathbf{0}$ & No realizable & \\
N/A & No se aplica & \\
\hline
\end{tabular}

Adicionalmente, el instrumento MHAVIDA evalúa la satisfacción con la realización de los hábitos de vida de la persona en CD con una escala tipo Likert, que oscila entre 1 y 5 . Esta evaluación puede aumentar las opciones y expectativas de la persona en CD. Sin embargo, los resultados de esta escala no se tienen en cuenta en el cálculo de la puntuación.

Tabla 3. Escala de calificación del nivel de satisfacción

\begin{tabular}{cl}
\hline Puntuación & Nivel de satisfacción \\
\hline $\mathbf{5}$ & Muy satisfecho \\
$\mathbf{4}$ & Satisfecho \\
$\mathbf{3}$ & Más o menos satisfecho \\
$\mathbf{2}$ & Insatisfecho \\
$\mathbf{1}$ & Muy insatisfecho \\
\hline
\end{tabular}

Según la Resolución 008430 del 4 de octubre de 1993 del Ministerio de Protección Social de Colombia, este estudio se consideró sin riesgo y fue aprobado por el Comité de Investigaciones de la Universidad de Santander. Se solicitó consentimiento institucional a la Fundación FANDIC, e informado por escrito a las madres o cuidadores de los niños y niñas de esta fundación.
Se aplicaron medidas de tendencia central y dispersión, de posición o porcentajes, según la naturaleza y distribución de las variables. También se realizó una descripción narrativa de los cambios realizados en cada versión del instrumento MHAVIDA, tanto en la fase de adaptación cultural como en la fase de validez facial.

\section{Resultados}

La adaptación cultural se realizó mediante la aplicación del instrumento a 6 madres de niños y niñas sin CD de la comuna 2 de Bucaramanga. Las edades de estas madres oscilaban entre 20 y 25 años. En el cuestionario se modificó el inicio de las preguntas y se cambió el verbo, de participio a presente simple. De las 64 preguntas del instrumento, se modificaron 16 que correspondieron a los hábitos de vida: Nutrición $(n=1)$, condición corporal $(n=4)$, cuidado personal $(\mathrm{n}=3)$, desplazamientos $(\mathrm{n}=2)$, responsabilidades $(\mathrm{n}=2)$, vida comunitaria $(\mathrm{n}=2)$, educación $(\mathrm{n}=1) \mathrm{y}$ recreación $(n=1)$. Estas preguntas equivalen al $25 \%$ del total de preguntas.

Tabla 5. Preguntas modificadas a partir de la adaptación cultural del instrumento MHAVIDA para niños y niñas de 5 a 13 años

Modificación Versión 2.0

\section{Nutrición}

Selecciona los alimentos apropiados para sus comidas, según su gusto y su dieta (la cantidad, el tipo de comida, etc.)
Escoge las comidas según su gusto y dieta (cantidad, tipo de comida) 


\section{Pregunta según Versión 1.0}

\section{Condición corporal}

Entrar y salir de la cama

Durmiendo (conforme, duración, profundidad)

Compromiso en actividades físicas para el mantenimiento

o mejorar la salud física o estado de salud.

Comprometiendo en actividades ligeras que son relajadas o requieren atención (escuchando música o una historia, juegos de memoria, etc.)

\section{Cuidado personal}

Asistiendo a la higiene personal (lavando, cepillándose, peinándose el cabello, tomando baño o una ducha, etc.) Utiliza los equipos sanitarios de su casa (incluyendo el método o el equipo para evacuar el agua o el método de vaciamiento)

Hace parte del cuidado de su salud. (Primeros auxilios, medicación y recomendaciones terapéuticas, etc.)

\section{Desplazamientos \\ Monta una bicicleta (como medio de transporte, para el ocio, etc.) \\ Como pasajero en un vehículo (automóvil, autobús, taxi, etc.) (el transporte es considerado una adaptación)}

\begin{tabular}{l} 
Responsabilidad \\
Reconoce el valor de monedas y facturas, usándolos \\
correctamente \\
El cargo tomando de él, estando de pie a para corrige \\
\hline Vida comunitaria \\
\hline Participa en las actividades de grupos de la comunidad, las \\
asociaciones de estudiantes, etc. (scouts, comités de la \\
clase, varias organizaciones, etc.) \\
Se relaciona o participa en actividades religiosas o \\
espirituales
\end{tabular}

\section{Educación}

Hace parte de las actividades escolares (extra-curricular, excursiones, días de campo, etc.)

\section{Recreación}

Realiza actividades turísticas (viajes, visita a sitios naturales o históricos, etc.)

La Validez Facial por parte de las madres de niños y niñas en $\mathrm{CD}$, se realizó en 19 de ellas, cuyos hijos o hijas asisten a la Fundación FANDIC. El rango de edad de las participantes fue de 18 a 45 años y el promedio de edad de sus hijos de 9,5 $\pm 2,54$. En éstos, predominó el diagnóstico de parálisis cerebral $(31,8 \%)$. Todas las madres pertenecían a los estratos 1 y 2 de la Comuna 2 de Bucaramanga, y su nivel educativo no superaba la educación secundaria.

Como resultado de esta fase, el testigo encargado de registrar las preguntas, sugerencias o comentarios de las madres encontró que la expresión"centro de cuidado diario" era confusa para las participantes, por

\section{Modificación Versión 2.0}

Se acuesta y se levanta de la cama

Duerme confortablemente y en el tiempo adecuado.

Realiza actividades físicas para mantener o mejorar su salud física o estado de salud.

Realiza actividades lúdicas que relajan o requieren de su atención (escuchar música, una historia, juegos de memoria, etc.)

Participa en su higiene corporal (lavarse la cara, cepillarse los dientes, peinarse el cabello, tomar una ducha, etc.)

Utiliza el inodoro de la casa (incluyendo métodos de bajar el agua)

Participa en el cuidado de la salud personal (primeros auxilios, medicación y recomendaciones terapéuticos)

Monta bicicleta, patineta o patines

Usa un vehículo como pasajero (carro, bus, taxi, etc.) (el transporte es considerado una adaptación)

Reconoce el valor del dinero y usa las monedas y billetes correctamente

Se hace cargo de sí mismo, defiende sus derechos

Se relaciona o participa en actividades de la comunidad, asociaciones estudiantiles, etc. (investiga, comités de clase, organizaciones, etc.)

Participa en actividades religiosas o actividades espirituales
Participa en actividades escolares (extra-curricular, excursiones, días del campo, etc.)

Participa en actividades turísticas (viaja, visita sitios naturales o históricos, etc.) lo cual se modificó a centro de educación temprana. Asimismo, hubo confusión en la pregunta "utiliza las instituciones de deportes locales...", la cual fue cambiada por "Utiliza las instalaciones deportivas...".

En la pregunta:" ¿come en un restaurante (servicio a la mesa o comida rápida)", las madres no tenían claro si la pregunta se refería a la acción de ir al restaurante o comer en el restaurante. Y en la pregunta:" ¿utiliza los servicios prestados por clínicas, hospitales y centros de rehabilitación?", las madres no tenían claro si se estaba preguntando si ellas llevaban al niño a los servicios de salud, si contaban con servicios de salud o si la mamá usa los servicios de salud. Por lo anterior,

Cienc. innov. salud. Junio 2013; 1 (1):18 - 26. Universidad Simón Bolívar (Col). ISSN: 2344-8636 
se decidió crear un manual en el que se registró la forma de realizar las preguntas y a qué se refiere cada una de ellas. Este manual se puede solicitar a las autoras, previa autorización de uso de los creadores del instrumento.

Como sugerencias, las madres manifestaron que en cada pregunta se debería mencionar el sujeto que

Tabla 7. Preguntas del instrumento MHAVIDA. Versión en Español 3.0 según grupo de edad

Instrumento MHAVIDA para niños, niñas y jóvenes de 5 - 13 años

Nutrición

1. El niño(a)/joven escoge las comidas según su gusto (cantidad, tipo).

2. El niño(a)/joven prepara sus comidas (incluyendo el uso de ciertos aparatos electrodomésticos).

3. El niño(a)/joven come sus alimentos (incluyendo el uso de platos y utensilios, buenos modales de la mesa, etc.).

4. El niño(a)/joven come por fuera en un restaurante (servicio a la mesa y comidas rápidas).

\section{Condición corporal}

5. El niño(a)/ joven se puede acostar y levantar de la cama.

6. El niño(a)/joven duerme confortablemente y en el tiempo adecuado.

7. El niño(a)/ joven realiza actividades físicas para mantener o mejorar su salud física o estado de salud.

8. El niño(a)/joven realiza actividades lúdicas que relajan o requieren de su atención (escuchar música, una historia, juegos de memoria, etc.).

\section{Cuidado personal}

9. El niño(a)/joven participa en su higiene corporal (lavarse la cara, cepillarse los dientes, peinarse el cabello, tomar una ducha, etc.).

10. El niño(a)/joven utiliza el inodoro de la casa (incluyendo el método de bajar el agua).

11. El niño(a)/joven utiliza inodoros diferentes a los de su casa (incluyendo el método de bajar el agua).

12. El niño(a)/joven se viste y se desviste de la cintura hacia arriba (camisa, botones, cremallera, escoge la ropa y accesorios).

13. El niño(a)/joven se viste y se desviste de la cintura hacia abajo (pantalones, botones, cremalleras, cordones, escoge la ropa y accesorios)

14. El niño(a)/joven se pone, quita y hace mantenimiento de sus ayudas técnicas, (prótesis, sillas de ruedas, audífono, lentes del contacto, gafas, etc.).

15. El niño(a)/joven participa en el cuidado de la salud personal (primeros auxilios, medicación y recomendaciones terapéuticos).

16. El niño(a)/joven utiliza los servicios ofrecidos por una clínica, hospital, centro de rehabilitación o puesto de salud de su comunidad.

\section{Comunicación}

17. El niño(a)/joven se comunica con un adulto en la casa o en la comunidad (expresa sus necesidades, mantiene una conversación, etc.)

18. El niño(a)/joven se comunica con personas jóvenes en la casa o en la comunidad (expresa sus necesidades, mantiene una conversación, etc.)

19. El niño(a)/joven se comunica con un grupo de personas en casa o en la comunidad (expresa sus ideas, mantiene una conversación, etc.).

20. El niño(a)/joven se comunica por escrito (escribe palabras, frases, texto corto, etc.).

21. El niño(a)/joven lee y entiende la información escrita (periódicos, libros, cartas).

22. El niño(a)/joven utiliza el teléfono de la casa.

23. El niño(a)/joven utiliza un computador.

24. El niño(a)/joven utiliza el televisor, una cámara, el radio, mp3.

Vivienda

25. El niño(a)/joven participa en las tareas de limpieza de la casa (limpieza, tender la cama, ordenar, etc.)

26. El niño(a)/joven participa en el mantenimiento del exterior de su casa (pasto, tierra, jardín, etc.).

27. El niño(a)/joven entra y sale de la casa y se desplaza dentro de la casa.

28. El niño(a)/joven se mueve alrededor y dentro de la casa.

29. El niño(a)/joven usa los muebles y los equipo de la casa (la mesa, organizador, lámparas, objetos).

30. El niño(a)/joven se desplaza fuera de la casa (verja, patio, jardín).

Desplazamientos

31. El niño(a)/joven se desplaza en las calles y aceras (incluso cruzar la calle).

32. El niño(a)/jovense desplaza en las superficies resbaladizas o desiguales (agua, arena, piedras, pasto).

33. El niño(a)/joven monta bicicleta, patineta o patines.

34. El niño(a)/joven usa un vehículo como pasajero (carro, bus, taxi, etc.) (el transporte es considerado una adaptación).

Cienc. innov. salud. Junio 2013; 1 (1):18 - 26. Universidad Simón Bolívar (Col). ISSN: 2344-8636

http://portal.unisimonbolivar.edu.co:82/rdigital/innovacionsalud 


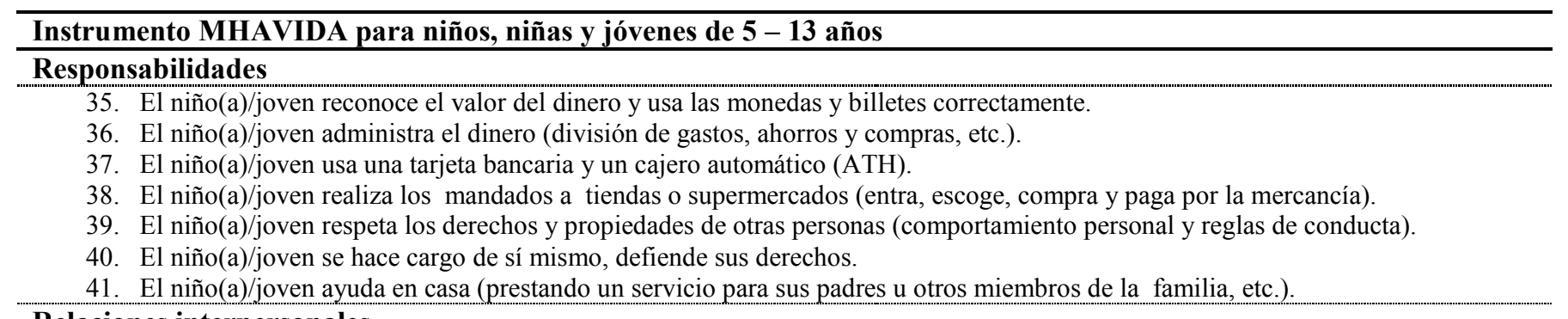

\section{Relaciones interpersonales}

42. El niño(a)/joven mantiene una relación afectiva con los padres.

43. El niño(a)/joven mantiene una relación afectiva con otros miembros de la familia cercana (hermanas, hermanos, etc.).

44. El niño(a)/joven mantiene relaciones afectivas con otros familiares (abuelos, primos, etc.).

45. El niño(a)/joven se relaciona o participa en actividades sexuales (información, discusiones, contacto físico, etc.).

46. El niño(a)/joven mantiene relaciones afectivas con sus compañeros (las actividades escolares, recreativas, etc.).

47. El niño(a)/joven mantiene relaciones sociales con los adultos (maestros, instructores, etc.).

\section{Vida comunitaria}

48. El niño(a)/joven se relaciona o participa en actividades de la comunidad, asociaciones estudiantiles, etc. (investigación, comités de clase, organizaciones).

49. El niño(a)/joven participa en actividades religiosas o espirituales

\section{Educación}

50. El niño(a)/joven llega, entra y se desenvuelve en la escuela (incluye el transporte escolar)

51. El niño(a)/joven participa en actividades de aprendizaje de la escuela (talleres, clases, tareas, exámenes, etc.).

52. El niño(a)/joven toma clases especializadas (educación física, música, baile, etc.).

53. El niño(a)/joven usa los medios escolares (cafetería, transporte escolar, patio de juegos, guardería, etc.).

54. El niño(a)/joven realiza las tareas.

55. El niño(a)/joven participa en actividades escolares extra-curriculares (excursiones, días del campo, etc.).

\section{Recreación}

56. El niño(a)/joven practica actividades deportivas o recreativas (deportes y juegos, recreación al aire libre, etc.).

57. El niño(a)/joven participa en los juegos individuales o de grupo dentro o al aire libre (juegos de mesa, juegos de pelota, videojuegos, etc.).

58. El niño(a)/joven asiste a actividades deportivas (futbol, baloncesto, etc.).

59. El niño(a)/joven participa en eventos culturales, artísticos o artesanales (música, baile, artes y danzas, etc.).

60. El niño(a)/joven asiste a eventos artísticos o culturales (conciertos, películas, teatro, etc.).

61. El niño(a)/joven participa en actividades turísticas (viaja, visita sitios naturales o históricos, etc.).

62. El niño(a)/joven llega, entra y se mueve en instalaciones recreativas, locales y culturales (Comfenalco, recrear, bibliotecas).

63. El niño(a)/joven utiliza las instalaciones culturales y recreativas locales (Comfenalco, recrear, bibliotecas).

Trabajo

64. El niño(a)/joven realiza pequeños trabajos con pago o sin pago de dinero (cuida niños menores, ayuda en una tienda, deshierba pasto).

\section{DISCUSIÓN}

El instrumento de medición de hábitos de vida MHAVIDA para el rango de 5-13 años de edad, que evalúa participación social, fue traducido oficialmente $\mathrm{y}$ adaptado al lenguaje regional con preguntas comprensibles, claras y fáciles de responder. Por lo anterior, se obtuvo un instrumento con validez facial y una adaptabilidad cultural pertinente para el medio.

Este instrumento evalúa la participación, la cual constituye uno de los cuatro dominios del componente de discapacidad y funcionamiento de la CIF (1). Es de resaltar que la CIF agrupa en un solo código los términos actividades y participación, aunque son dos constructos diferentes. Las actividades son de orden individual y las personas las realizan por sí mismas; mientras que la participación se enfoca en el desarrollo de la persona en $\mathrm{CD}$ en la sociedad y se realiza con otras personas. Sin embargo, actividades como pensar, comer y viajar, que se realizan de manera independiente, son consideradas aspectos de la participación, lo cual indica que actualmente existe debate sobre los aspectos que debe evaluar la participación. Este último es un campo relativamente nuevo, pero al mismo tiempo es relevante para las personas en $\mathrm{CD}$, los miembros de su familia, la sociedad y, sin duda, para el equipo rehabilitador (8).

La taxonomía para actividades y participación de la CIF incluye 9 dominios: Aprendizaje y aplicación del conocimiento, tareas y demandas generales, 
comunicación, movilidad, autocuidado, vida doméstica, interacciones y relaciones interpersonales, áreas principales de la vida y vida comunitaria, social y cívica. Según Resnik (8),el instrumento MHAVIDA, versión en inglés 3.1 con 77 ítems, cubre los anteriores dominios y, adicionalmente, contiene 13 preguntas que indagan por aspectos no considerados como participación en la CIF.

Los hallazgos del presente estudio muestran que el instrumento MHAVIDA cuenta con preguntas bien redactadas y comprensibles para las madres de los niños y niñas en $C D$. Esto es esencial porque ellas son quienes tienen más expectativas sobre el desarrollo de sus hijos en la sociedad, saben qué actividades pueden o no realizar y, por lo tanto, sus respuestas se aproximan a la real participación de sus hijos.

Estudios previos han evaluado la validez del instrumento MHAVIDA en otros idiomas. Por ejemplo, Fougeroyas (5) evaluó la validez de contenido del instrumento MHAVIDA en su versión original (248 preguntas) en idioma Francés. Fue realizada por 12 expertos de diferentes áreas y como consecuencia se eliminaron algunas preguntas y se modificaron otras. Además, la escala de puntuación fue mejorada e invertida, indicando mayor cumplimiento del hábito de vida cuando el puntaje se incrementa (versión en Francés 2.0). Igualmente, los expertos concluyeron que el instrumento MHAVIDA cubría la mayoría de hábitos de vida que evalúan participación social. En otro estudio, Lemmens (9) encontró una buena validez discriminante al comparar los resultados de la aplicación del instrumento MHAVIDA versión holandesa en dos grupos, uno de adultos mayores con enfermedades crónicas, y el otro con adultos saludables. Asimismo, se encontró una muy buena validez convergente al comparar los resultados del instrumento MHAVIDA y el Cuestionario de Impacto sobre la Participación y Autonomía y la Escala de Discapacidad de Londres.

Por su parte, Noreau (6) evaluó las propiedades psicométricas del instrumento MHAVIDA, versión en inglés, en niños y niñas de 5 a 13 años. En este caso, la validez de contenido del instrumento fue evaluada por un panel de expertos que incluía padres de niños y niñas con limitaciones funcionales, clínicos pediátricos experimentados e investigadores. La mayoría (67$90 \%$, dependiendo de la dimensión) juzgó como adecuada la representatividad de los ítems para evaluar las diferentes dimensiones de la participación social.
Cabe destacar que en el estudio anterior un elevado porcentaje de padres de familia consideraron que las preguntas de movilidad, vida comunitaria y comunicación representaban adecuadamente la participación social de sus hijos; mientras que una baja proporción tuvo esta misma apreciación para las categorías educación y recreación (6). Estos resultados coinciden en parte con los del presente estudio, en el cual algunos términos empleados en la categoría educación fueron confusos para las madres. Otra categoría con problemas de comprensión fue la de nutrición.

Como limitación se debe considerar que el instrumento fue aplicado por estudiantes de Fisioterapia, quienes tienen menos experiencia en entrevistas y en el área de discapacidad, comparados con los fisioterapeutas propiamente dichos, quienes, por contar con más experiencia, pueden recolectar información más confiable. En cuanto al uso del instrumento, su aplicación fue autorizada por los creadores con fines de investigación; por lo tanto, los derechos de uso en la práctica clínica deben ser adquiridos

En definitiva, mediante este estudio se obtuvo un instrumento útil para evaluar la participación social a partir de los hábitos de vida de la población infantil con discapacidad, lo cual proporciona una base sólida para mejorar las intervenciones en rehabilitación. Además, el instrumento puede ser utilizado e implementado en investigaciones futuras, que involucren niños y niñas en CD entre 5 y 13 años en cualquier contexto regional de Colombia.

\section{Referencias}

1. Organización Mundial de la Salud. CIF: Clasificación Internacional del Funcionamiento, de la Discapacidad y de la Salud. OMS/OPS; 2001.

2. Departamento Administrativo Nacional de Estadística DANE. Base de Datos del Registro de Localización y Caracterización de Personas con Discapacidad. Bogotá (DC): Dirección de Censos y Demografía; 2005-2006.

3. Fougeyrollas PN, St-Michel G. Guide de 1.utilisateur «Instrument de mesure des habitudes de vie » (MHAVIDA 2.1) et «Instrument de 
mesure de la qualité de l.environnement ». Réseau International CIDIH 1997;9(1):14.

4. Atte A, Haley SM, Kooyoomjian JT. Are the ICF Activity and Participation dimensions distinct? J Rehabil Med. 2003;35(3):145-149.

5. Fougeyrollas PN, Bergeron $\mathrm{H}$, Cloutier R, Dion SA, St-Michel G. Social consequences of long term impairments and disabilities: conceptual approach and assessment of handicap. Int $\mathbf{J}$ Rehabil Res. 1998;21(2):129-141.

6. Noreau L, Lepage C, Boissiere L, Picard R, Fougeyrollas P, Mathieu J, et al. Measuring participation in children with disabilities usingthe Assessment of Life Habits. Dev Med Child Neurol. 2007;49(9):666-671.

7. Orozco L. Medición en salud: Diagnóstico y Evaluación de Resultados, un manual crítico más allá de lo básico: Medición o de como se hacen metros. Bucaramanga: División de publicaciones UIS; 2010.

8. Resnik L, Plow MA. Measuring participation as defined by the international classification of functioning, disability and health: an evaluation of existing measures. Arch Phys Med Rehabil. 2009;90(5):856-866.

9. Lemmens J, ISM van Engelen E, Post MW, Beurskens AJ, Wolters PM, de Witte LP. Reproducibility and validity of the Dutch Life Habits Questionnaire (LIFE-H 3.0) in older adults. Clin Rehabil. 2007; 21(9):853-862. 